\title{
Barbie Doll Forehead: CAD Design from Silicon Block
}

\author{
Shruti Marwah ${ }^{1}$ Arjun Handa ${ }^{1}$ \\ ${ }^{1}$ Department of Plastic Surgery, Handa Nursing Home, New Delhi, \\ Address for correspondence Dr. Shruti Marwah, MCh, Department of \\ India \\ Indian J Plast Surg 2022;55:111-114.

\begin{abstract}
Plastic Surgery, Handa Nursing Home, 20 Raja Garden, New Delhi
\end{abstract} \\ 110015, India (e-mail: shrutim150@gmail.com).
}

\author{
Abstract \\ Keywords \\ - facial feminization \\ - forehead \\ augmentation \\ - silicone implants
}

The forehead is an important determinant of masculine or feminine look of the face. There are many methods described to achieve forehead augmentation for a more feminine face, and they include fillers, autologous fat grafting, silicone implant or methyl methacrylate. We present a case of a "Barbie doll forehead," which is an interesting trend in Asian countries. Availability of silicone implants of various sizes are not uniform and currently unavailable in India. Alternatively, we devised carved silicone block using computer-aided design (CAD). We also discuss the various methods described for forehead augmentation with their advantages and disadvantages.

\section{Introduction}

Numerous procedures are described to achieve facial feminization, and forehead contouring, and augmentation is one of them. ${ }^{1}$ The case we present here is a transsexual female who had already undergone multiple procedures elsewhere for feminization.

The "Barbie doll forehead" is a commonly performed procedure in countries like Korea and pertains to forehead augmentation.

Therefore, these countries have customized implants in various sizes, ranging from small and medium to large. However, no such trend exists in India, and due to COVID, we were unable to source the implant from other countries. Here, we describe how with the use of 3D printing and the aid of a sculptor, we were able to achieve the final result.

Although this article concentrates on the aspect of forehead augmentation, we also performed a malpositioned chin implant removal with T-osteotomy chin genioplasty to achieve overall facial feminization. We also performed malpositioned breast implant removal, correction of implant pocket, and exchange with new silicone implant.

published online

February 25, 2022
DOI https://doi.org/ 10.1055/s-0041-1740524. ISSN 0970-0358.

\section{Methodology}

\section{Preoperative Preparation}

Clinical pictures were taken, and 3D reconstruction of noncontrast computed tomography (NCCT) of head and face was done ( - Fig. 1). The computer-aided design (CAD) was used to choose the appropriate size of implant, such that its lateral extent was till bilateral superior temporal fusion lines, inferior extent was till supraorbital bridge, and superior extent was $2 \mathrm{~mm}$ beyond the hair line. A negative template was fabricated using 3D printing. It was then used to manufacture a positive template with industrial grade silicon (-Figs. 2-3) A silicone block (medical grade with durometer 20 and size $15.5 \times 7.5 \times 1.3 \mathrm{~cm})$ and the template were handed over to a sculptor to carve out the exact replica with appropriate dimensions $(6 \mathrm{~mm}$ thickness in the center, i.e., at the level of the most prominent part of the forehead, with tapered ends). Once the implant was received, it was autoclaved and made sterile.

\section{Operative Steps}

Patient was positioned supine under general anesthesia. The bilateral temporal fusion lines (lateral extent of the pocket) (c) 2022. Association of Plastic Surgeons of India. All rights reserved. This is an open access article published by Thieme under the terms of the Creative Commons Attribution-NonDerivative-NonCommercial-License, permitting copying and reproduction so long as the original work is given appropriate credit. Contents may not be used for commercial purposes, or adapted, remixed, transformed or built upon. (https://creativecommons.org/ licenses/by-nc-nd/4.0/)

Thieme Medical and Scientific Publishers Pvt. Ltd., A-12, 2nd Floor, Sector 2, Noida-201301 UP, India 

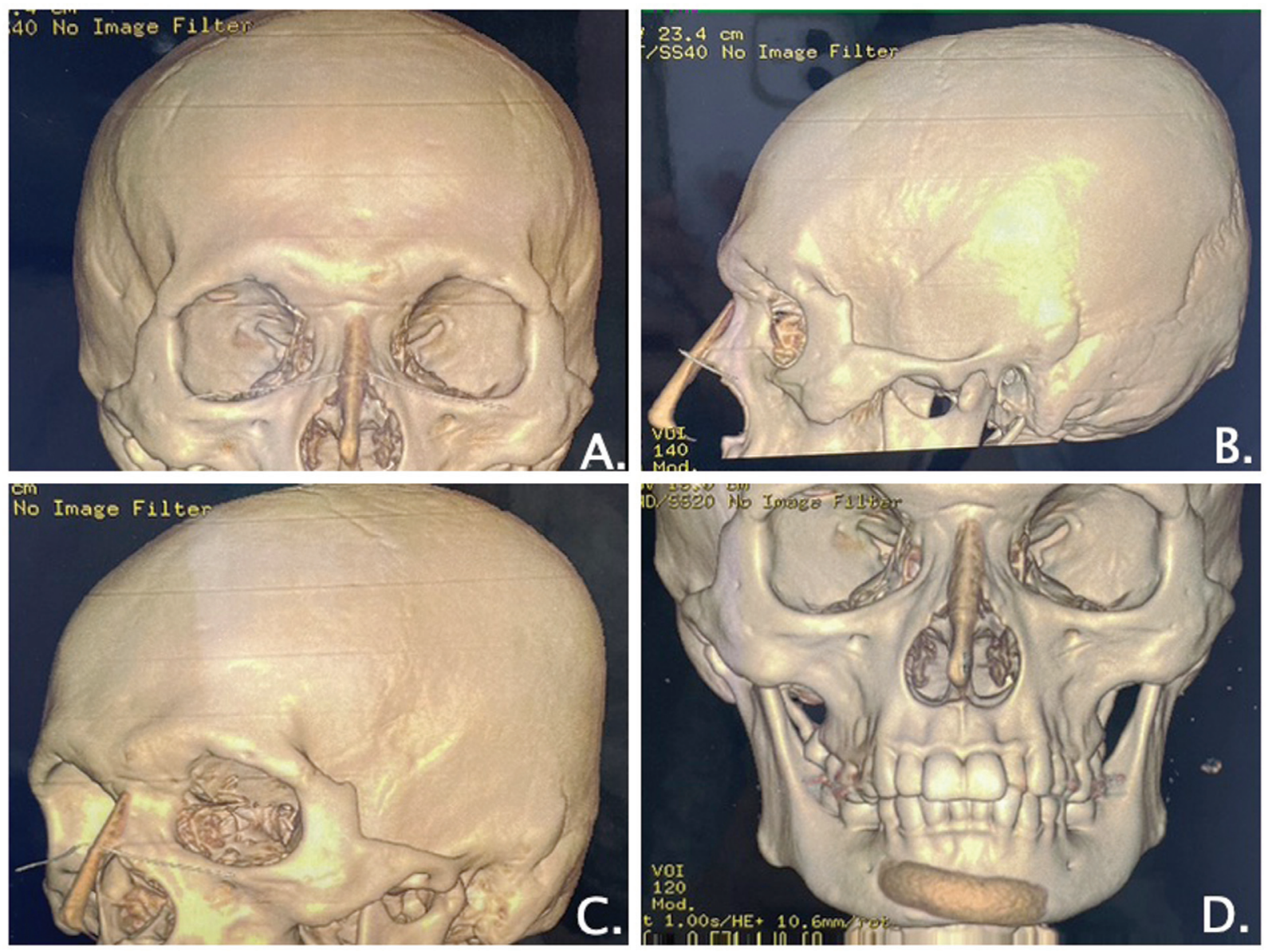

Fig. 1 3D reconstruction of noncontrast computed tomography (NCCT) head and face which will be used for computer-aided design (CAD). (A). Front view. (B). Side view. (C). Oblique view. (D). Front view of the face showing the silicone nasal implant and malpositioned silicone chin implant (placed elsewhere).

and supraorbital ridge (inferior extent of the pocket) were marked, a $5 \mathrm{~cm}$ incision was made 3 finger breadths behind the hairline, and subperiosteal flap was elevated using special forehead elevators (-Fig. 4). Implant was inserted into the tight pocket, and incision was closed in two layers with 3-0 vicryl and 3-0 nylon.

\section{Post-operative Management}

A firm compressive dressing was applied for 24 hours, and patient was asked to lie down in head elevation for 5 days. Sutures were removed at 10 days. Postop period was uneventful. (-Fig. 5).

\section{Discussion}

Forehead augmentation can be achieved by soft-tissue or skeletal augmentation. The various methods for skeletal augmentation include alloplastic material such as silicone implant, methyl methacrylate (MMA), or expanded polytetrafluoroethylene (PTFE). ${ }^{2}$ Soft-tissue augmentation can be achieved by autologous fat grafting or fillers. ${ }^{3,4}$

A rounded, protruding, and fuller forehead is considered more attractive and feminine, as opposed to a flat forehead, especially in the Asian population., ${ }^{2,5}$
Therefore, the term pertains to the typical smooth, rounded, and protruded forehead similar to a Barbie doll, albeit in an exaggerated form. Although it is an uncommon reference in the Indian scenario, but in the South Asian and Korean population, Barbie doll forehead reference is quite commonly used to indicate the feminization of the forehead with augmentation.

Silicone is one of the most commonly used methods to achieve forehead augmentation. The advantages are that silicone is an inert material, can be sculpted into the desired shape, does not require an additional donor site like fat, and is safe. However, there is a chance that despite accurate molding, the edges of the implant may be visible if a gap exists between the implant and forehead bone. ${ }^{6}$

Park in his article described two cases, ${ }^{6}$ where he utilized alginate to create negative and positive mold of the forehead and then got the implant carved. ${ }^{7}$ We attempted to do the same; however, the resulting template was unsatisfactory and hence was discarded.

In the second case, he describes use of cone-beam computed tomography (CBCT) and then creation of a rapid prototype (RP) model; further technique has not been elaborated. This is similar to our method, except that we used 


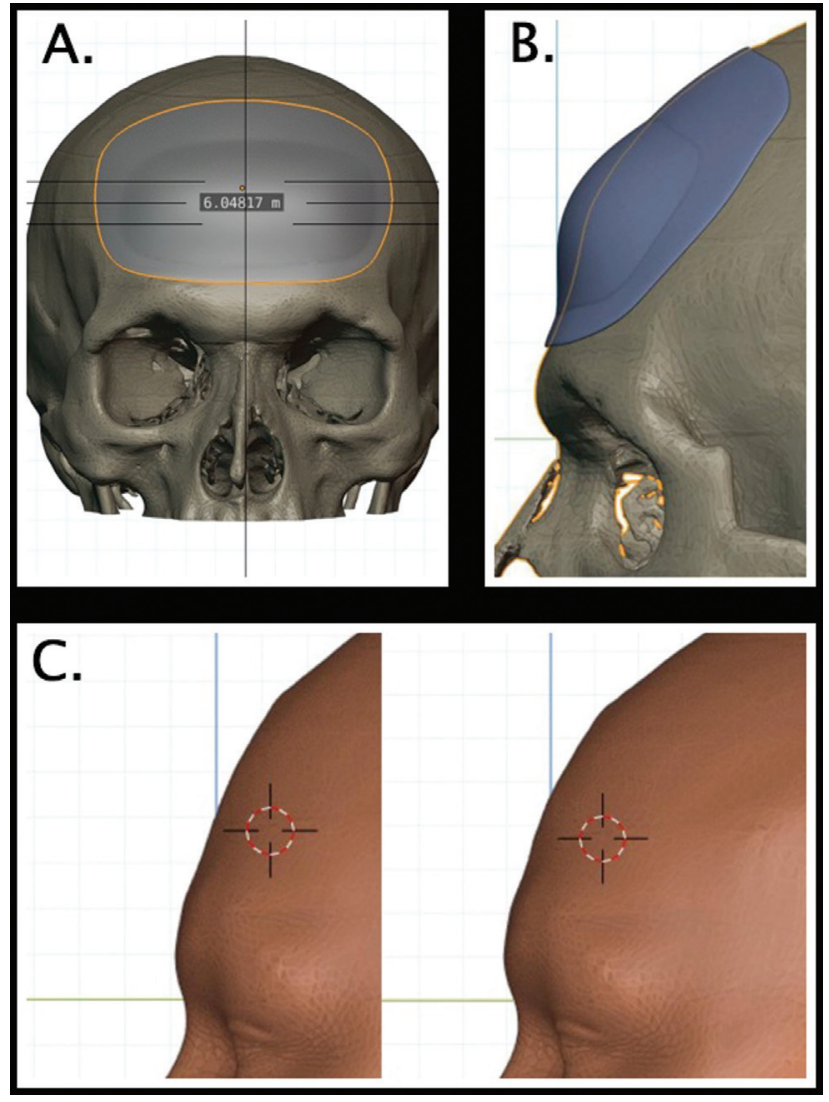

Fig. 2 Computer-aided design (CAD). (A). Frontal view showing the implant with $6 \mathrm{~mm}$ thickness in the center. (B). Lateral view showing the implant in relation to the supra-orbital ridge and temporal line. (C). Soft-tissue reconstruction to show to the patient the expected change in shape.

NCCT scan with 3D printing of negative and positive template, and an industrial silicone template was used to help carve out the final implant. The task of CT scan and CAD as well as 3D printing was outsourced to various firms to achieve the final result as planned.

MMA has the advantage of being nonthermoconductive, the shape can be molded intraoperatively by addition or burring, and also it does not cause any tissue ingrowth which aids in removal later. However, increased operative time, the risk of injury due to an exothermic reaction, contact of fluid with blood, and material toxicity are all its disadvantages. ${ }^{8}$

Autologous fat grafting for facial contouring is another commonly used method, as it is minimally invasive and does not require use of any foreign body implants. However, it has the biggest disadvantage of unpredictable resorption and thus the need of multiple sessions. ${ }^{9}$

We opted for silicone implant after discussion with the patient. The use of CAD and 3D printing of the final implant has been described previously for MMA implants ${ }^{2}$ but not for silicone, as medical-grade flowable silicon is not available to formulate the implant.

Finally, the silicone block was handed to a sculptor who used burrs and special instruments to carve the final implant.
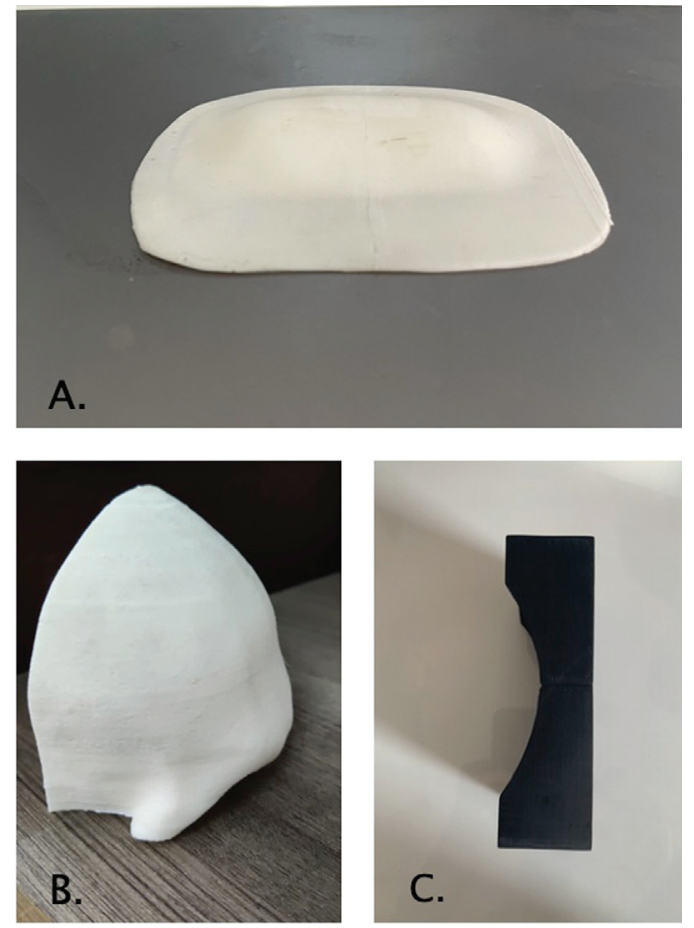

Fig. 3 (A) The positive template made from industrial grade silicone. (B) 3D printed replica of the patient's forehead. (C) Negative template which helped to carve out the implant.

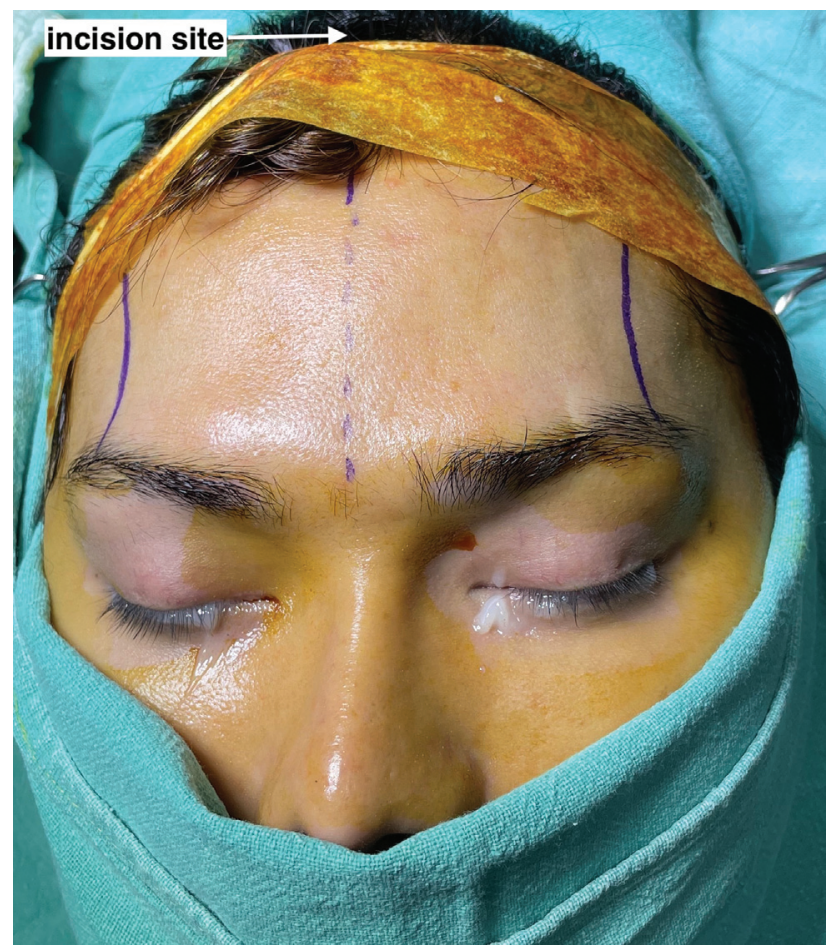

Fig. 4 Intraoperative view showing the marking of midline and bilateral temporal fusion lines, arrow showing incision site.

Although prefabricated silicone implants have been routinely available for different parts of face as well as body such as chin, cheeks and breasts, certain cases require customized implants for best results. 


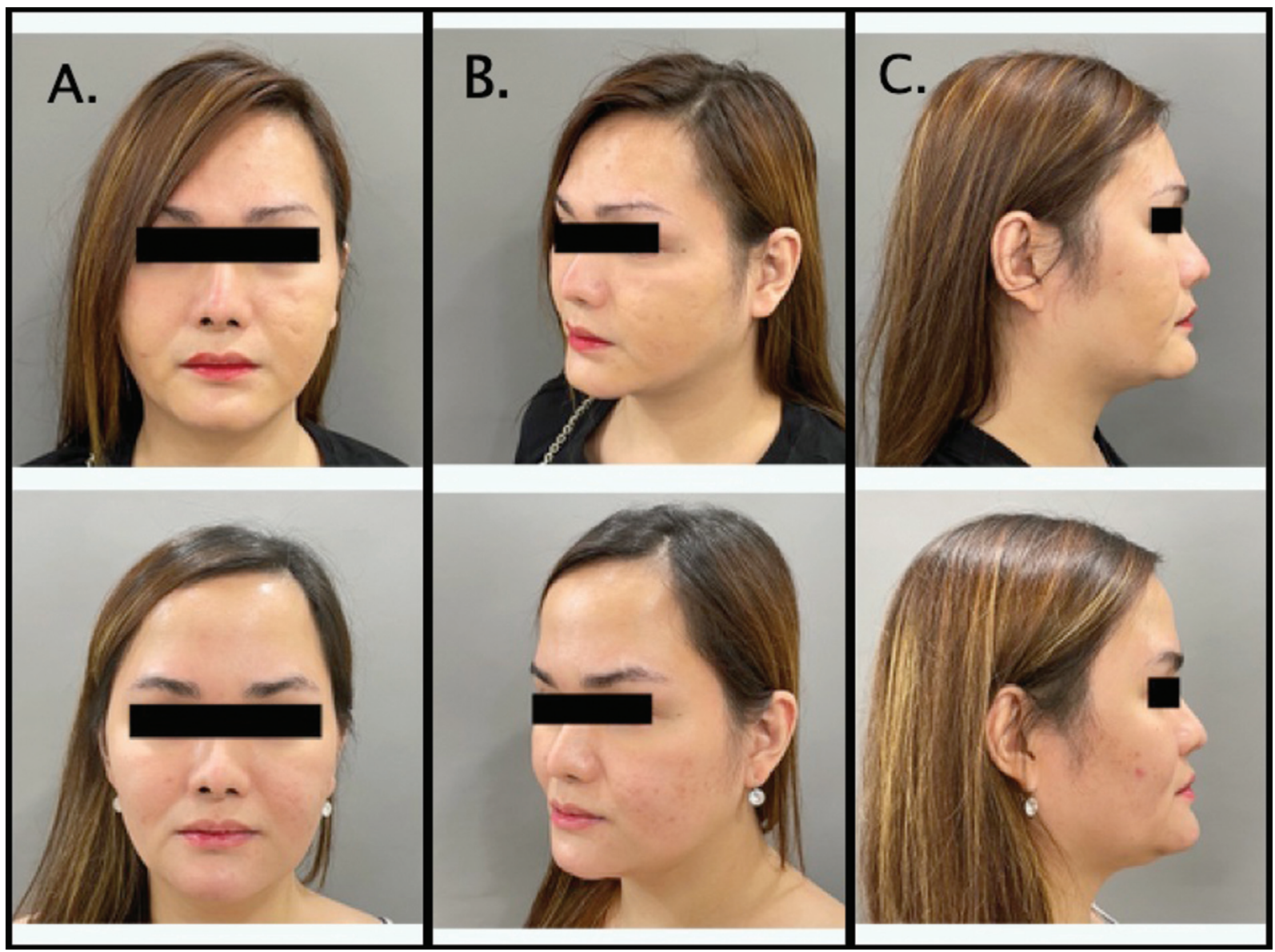

Fig. 5 Before and after pictures at 1 month. (A). Front view. (B). Left oblique view. (C). Right side view.

The help of a professional sculptor for carving of implants has been well-described, but it comes with the obvious disadvantage of the final result being dependent on the artist's hands, and minor imperfections may still result ${ }^{10}$ Hence, we attempted using a hybrid technique with both $3 \mathrm{D}$ printing as well as sculpting to achieve the best result possible.

The common complications associated with such procedures are infection, hematoma, buckling of the implant, and asymmetry or visible edges of the implant, but no such problems occurred in our case.

\section{Conclusion}

Forehead augmentation planning, as well as choice of appropriate method, can be tricky, although performing the procedure is simple and straightforward. Also, since our patient wanted a permanent result, without any additional donor site and minimal risk of intraoperative or postoperative complications, a silicone implant was a reliable and obvious choice.

\section{Conflict of Interest}

None declared.

\section{References}

1 Altman K. Facial feminization surgery: current state of the art. Int J Oral Maxillofac Surg 2012;41(08):885-894

2 Hirohi T, Nagai K, Ng D, Harii K. Integrated forehead and temporal augmentation using 3D printing-assisted methyl methacrylate implants. Aesthet Surg J 2018;38(11):1157-1168

3 Li X, Kubiak CA, Yang X, Kemp SWP, Cederna PS, Ma J. Forehead fat grafting: Asian facial contouring and augmentation. Plast Reconstr Surg 2019;144(05):1057-1065

4 Bass LS. Injectable filler techniques for facial rejuvenation, volumization, and augmentation. Facial Plast Surg Clin North Am 2015;23(04):479-488

5 Chao JW, Lee JC, Chang MM, Kwan E. Alloplastic augmentation of the Asian face: a review of 215 patients. Aesthet Surg J 2016;36 (08):861-868

6 Park YW. Frontal augmentation as an adjunct to orthognathic or facial contouring surgery. Maxillofac Plast Reconstr Surg 2016;38 (01):37

7 Wong JK. Forehead augmentation with alloplastic implants. Facial Plast Surg Clin North Am 2010;18(01):71-77

8 Park DK, Song I, Lee JH, You YJ. Forehead augmentation with a methyl methacrylate onlay implant using an injection-molding technique. Arch Plast Surg 2013;40(05):597-602

9 Sadeghi P, Sisti A. Forehead fat grafting: Asian facial contouring and augmentation. Plast Reconstr Surg 2020;146(04):499e-500e

10 Yang J, Shimek J, Salisbury M, Ersek RA. The art of custom silicone implants for difficult deformities. Aesthet Surg J 2010;30(05): $720-724$ 